\title{
A Precision Noise Spectral Density Comparator
}

\author{
C. M. Allred
}

(May 18, 1962)

\begin{abstract}
The theory is given for a precision comparator that measures the ratio of two noise spectral densities. The relative error of a single measurement is also derived. The comparator described removes or alleviates many of the problems in high-speed switching, and since the instrument operates under null conditions, the null position is essentially independent of amplifier noise and gain instabilities.
\end{abstract}

\section{Introduction}

The relative strength of two power sources or signal levels is often desired. As the strength of the two power sources decreases the determination of this ratio becomes increasingly difficult. When the desired signals have levels that are approximately equal or smaller than the internal noise level of a very low noise amplifier, the problems become severe. This is due not only to the masking effect of the internal amplifier noise but also to the instability of the amplifier gain. This in turn arises from the enormous amounts of gain needed.

The usual practice is to sample the outputs of the two levels at a switching rate that is high compared to the instability drift rate of the amplifier. An attenuator is usually used to reduce the level of the stronger signal to that of the other signal. The early pioneer of this method was the Dicke Radiometer [Dicke, 1946].

The switch with its noise, losses, and instabilities is often a limiting factor in such methods. The attenuator and the necessity of impedance matching is another problem source. These problems are eliminated or greatly alleviated by the method described herein.

\section{Principle of Operation}

The basic problem in such measurements is to make the amplifier gain drift essentially unimportant. In principle, the usual radiometer samples the two sources so that the variation of amplifier gain affects the amplified source levels of both sources equally. A preferable method would be to have both sources simultaneously present and no switching. The problem then, is to separate the two amplified sources at the output. If the two sources are statistically independent, they could be separated by correlation techniques. In principle this is what is done in the system described below.

Correlation techniques are an old tool and there are systems described in the literature that in various respects resemble the one discussed here [Fink, 1959; Freeman, 1958, p. 274].
The block diagram of the system is shown in figure 1. The individual components and also the configuration can take on different forms without changing the basic principles. The system described here is similar to one presently under construction.

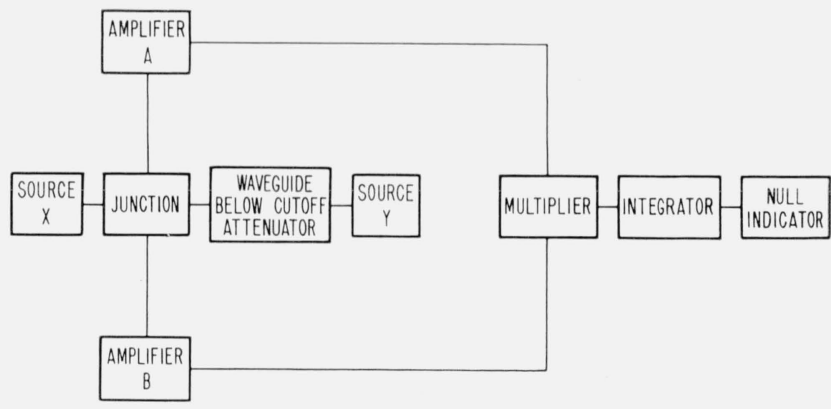

Figure 1. Block diagram of comparator.

\subsection{Sources}

The two sources may be very general. They may consist of random noise, pulse singals, CW, etc., or combinations of various types. It is assumed their output levels are constant during the measuring time.

\subsection{Attenuator}

For precison results the attenuator may be an accurate wave guide-below-cutoff (piston) attenuator. Small changes in attenuation may also be determined by measuring changes in the input level to the attenuator.

\subsection{Junction}

This simple but important component is shown in figure 2. It is a low-loss symmetrical coaxial $T$. Opposite to where the center leg joins the $T$, very small slits, perpendicular to the axis of the two side arms, are cut into the outer conductor. These are symmetrically placed on the $T$. The junction then is made an integral part of the attenuator so that the fields ( $\mathrm{TE}_{11}$ mode) within the attenuator guide impinge upon the slits of the $T$. In effect, the $T$ acts as a center-tapped secondary of a transformer. The source $X$ is applied to the center tap. 


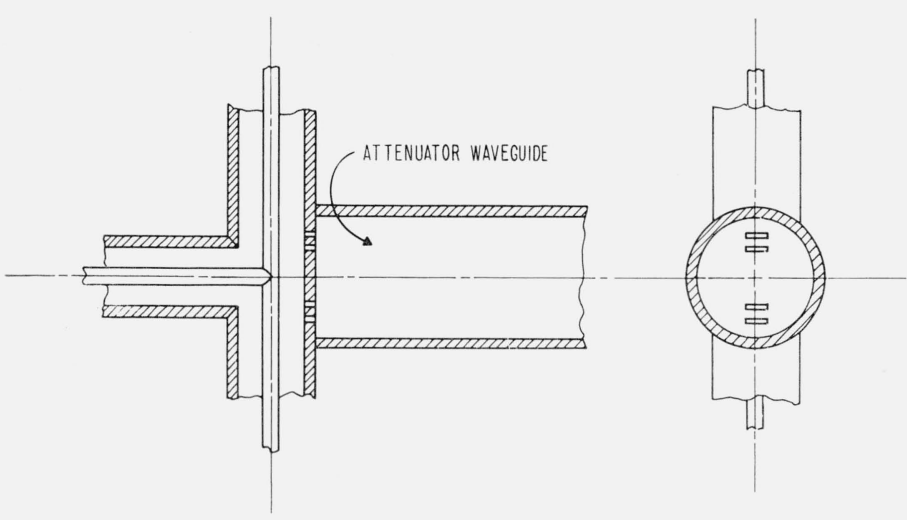

Figure 2. Junction.

\subsection{Amplifiers}

There is some value in having the two amplifiers similar, though this is not necessary. The amplifiers should be linear; at least the frequency components produced by the nonlinearity should be prevented from reaching the input to the multiplier. For some modes of system operation it is essential for the pass band characteristics of the amplifiers to remain constant during a measurement while the gain can vary. This is what primarily happens in an amplifier as the mutual conductance of a vacuum tube varies. The stability of the pass band can be enhanced by keeping the amplifier broadbanded except for stable band limiting devices.

\subsection{Multiplier, Filter, and Indicator}

The multiplier has the function of forming the product of the input voltages. The output of the multiplier, which is this product, is applied to the filter. The filter permits only the d-c and very low frequency components of this product to reach the indicator. Of course the filter and multiplier could be a single integral unit. The indicator responds to both positive and negative d-c voltages and is used only to obtain a null.

\section{General Theory}

Only the basic general theory will be presented. This describes, under certain assumptions, what the system measures and the expected error (due to the inevitable random fluctuations in the output) in this measurement. Possible errors due to various components deviating from the assumptions will be reserved for a forthcoming report when a specific system is being described.

The assumptions used in the following theory are:

1. The system, excluding the multiplier, is considered linear.

2. The amplifiers and filter, while linear, may be considered varying in time. The unit impulse response and Fourier system response functions of these units are then time variable. The analysis could have also been carried through with the assumption that the unit impulse functions were sample functions from a linearly independent ergodic process with the essential results being the same.

3. The signals present are all assumed to be sample functions from random processes that are bounded, and are singly and jointly ergodic as well as singly and jointly stationary. The periodic signals are considered to occur with a uniform random phase distribution over the ensemble.

4. The components, other than what is stated or implied above, are considered to be ideal. For example, the multiplier performs the proper product. Also, the junction is symmetrical, etc.

Sources $X$ and $Y$ respectively give rise to random signals $x(t)$ and $y(t)$ that are real time varying functions and contain, in general, both periodic and nonperiodic parts. The input to amplifier $\mathrm{A}$ is

$$
x(t)+y(t) .
$$

To amplifier $\mathrm{B}$, it is

$$
x(t)-y(t) .
$$

The output of amplifier $\mathrm{A}, e_{a}(t)$, is

$$
e_{a}(t)=\int_{-\infty}^{\infty} h_{a}(\alpha, t)\left[x(t-\alpha)+y(t-\alpha)+z_{a}(t-\alpha)\right] d \alpha
$$

where $h_{a}(\alpha, t)$ is the unit impulse response of amplifier $\mathrm{A}, z_{a}(t)$ is the internal noise of amplifier A referred to its input, and $\alpha$ is the variable of integration in the convolution integral. Likewise the output of amplifier $\mathrm{B}, e_{b}(t)$, is

$e_{b}(t)=\int_{-\infty}^{\infty} h_{b}(\beta, t)\left[x(t-\beta)-y(t-\beta)+z_{b}(t-\beta)\right] d \beta$

where the $b$ subscripts refer to amplifier B. The quantities $e_{a}(t)$ and $e_{b}(t)$ are multiplied together by means of the multiplier. The expected value or statistical average of the output of the multiplier. indicated by $\mathrm{E}\left(e_{a} e_{b}\right)$ or $\overline{e_{a} e_{b}}$ is

$$
\begin{gathered}
E\left(e_{a} e_{b}\right)=E\left[\left(\int_{-\infty}^{\infty} h_{a}(\alpha, t)[x(t-\alpha)\right.\right. \\
\left.\left.\quad+y(t-\alpha)+z_{a}(t-\alpha)\right] d \alpha\right) \\
\left.\left(\int_{-\infty}^{\infty} h_{b}(\beta, t)\left[x(t-\beta)-y(t-\beta)+z_{b}(t-\beta)\right] d \beta\right)\right] .
\end{gathered}
$$

Since $h_{a}(\alpha, t)$ and $h_{b}(\beta, t)$ are considered to be nonrandom functions,

$$
\begin{gathered}
E\left(e_{a} e_{b}\right)=\int_{-\infty}^{\infty} \int_{-\infty}^{\infty} h_{a}(\alpha, t) h_{b}(\beta, t) E([x(t-\alpha)+y(t-\alpha) \\
\left.\left.+z_{a}(t-\alpha)\right]\left[x(t-\beta)-y(t-\beta)+z_{b}(t-\beta)\right]\right) d \alpha d \beta
\end{gathered}
$$

where the order of integration and averaging have 
been interchanged. The expected value or statistical mean of the indicator output, $\vec{I}$, is given by

$$
\begin{array}{r}
\bar{I}=H_{d}(0, t) \int_{-\infty}^{\infty} \int_{-\infty}^{\infty} h_{a}(\alpha, t) h_{b}(\beta, t) E([x(t-\alpha) \\
\left.+y(t-\alpha)+z_{a}(t-\alpha)\right][x(t-\beta) \\
\left.\left.\quad-y(t-\beta)+z_{b}(t-\beta)\right]\right) d \alpha d \beta
\end{array}
$$

where $H_{d}(0, t)$ is the zero frequency response of the filter and indicator.

The expected value of the time varying signal function in (6) is composed of the statistical autocorrelation and cross correlation functions of the various random signals present. Thus

$$
E[x(t-\alpha) x(t-\beta)] \equiv R_{x}(\beta-\alpha)
$$

where $R_{x}(\beta-\alpha)$ is the statistical autocorrelation function of $x(t)$. Similarly

$$
E[x(t-\alpha) y(t-\beta)] \equiv R_{x y}(\beta-\alpha)
$$

with similar results for other combinations, where $R_{x y}(\beta-\alpha)$ is the cross correlation function of the functions $x(t)$ and $y(t)$.

From the foregoing, (6) may be written

$$
\begin{gathered}
\bar{I}=H_{d}(0, t) \int_{-\infty}^{\infty} \int_{-\infty}^{\infty} h_{a}(\alpha, t) h_{b}(\beta, t)\left[R_{x}(\beta-\alpha)\right. \\
-R_{y}(\beta-\alpha)-R_{x y}(\beta-\alpha)+R_{x z_{b}}(\beta-\alpha) \\
+R_{y x}(\beta-\alpha)+R_{y z_{b}}(\beta-\alpha)+R_{z_{a} x}(\beta-\alpha) \\
\left.-R_{z_{a} y}(\beta-\alpha)+R_{z_{a^{z} b}}(\beta-\alpha)\right] d \alpha d \beta .
\end{gathered}
$$

If the respective signals are uncorrelated all cross correlation functions become zero, and (8) reduces to (correlation between $z_{a}(t)$ and $z_{b}(t)$ is discussed later)

$$
\begin{aligned}
\bar{I}=H_{d}(0, t) \int_{-\infty}^{\infty} \int_{-\infty}^{\infty} h_{a}(\alpha, t) h_{b}(\beta, t) \\
\quad\left[R_{x}(\beta-\alpha)-R_{y}(\beta-\alpha)\right] d \alpha d \beta .
\end{aligned}
$$

The above correlation functions are related to the spectral densities of the various signals. Let $S_{x}(f)$ represent the spectral density of the signal $x(t)$. Then $S_{x}(f)$ and $R_{x}(\tau)$ are related (by definition) through the Fourier transform pair

$$
\begin{aligned}
& S_{x}(f)=\int_{-\infty}^{\infty} R_{x}(\tau) e^{-j 2 \pi f \tau} d \tau \\
& R_{x}(\tau)=\int_{-\infty}^{\infty} S_{x}(f) e^{j 2 \pi f \tau} d f
\end{aligned}
$$

Substituting the above values for the correlation functions into (9) together with the relation $\tau=\beta-\alpha$ yields

$$
\begin{array}{r}
\bar{I}=H_{d}(0, t) \int_{-\infty}^{\infty} \int_{-\infty}^{\infty} \int_{-\infty}^{\infty} h_{a}(\alpha, t) e^{-j 2 \pi f \alpha} h_{b}(\beta, t) e^{j 2 \pi f \beta} \\
{\left[S_{x}(f)-S_{y}(f)\right] d f d \alpha d \beta .}
\end{array}
$$

Now the impulse response function, $h_{a}(\alpha, t)$, is related to the complex frequency response function, $H_{a}(j 2 \pi f, t)$ by

$$
\int_{-\infty}^{\infty} h_{a}(\alpha, t) e^{-j 2 \pi f \alpha} d \alpha=H_{a}(j 2 \pi f, t)=\left|H_{a}(j 2 \pi f, t)\right| e^{j \theta_{a}}
$$

where $H_{a}(j 2 \pi f, t)$ is the complex frequency gain function of amplifier $\mathrm{A}$ and $\theta_{a}$ is the associated phase angle. Similarly

$$
\int_{-\infty}^{\infty} h_{b}(\beta, t) e^{j 2 \pi f \beta} d \beta=H_{b}^{*}(j 2 \pi f, t)=\left|H_{b}(j 2 \pi f, t)\right| e^{-j \theta_{b}} .
$$

In general, $\theta_{a}$ and $\theta_{b}$ are functions of time, though for simplicity in notation they are not explicitly written as such. Thus (11) becomes

$$
\begin{array}{r}
\bar{I}=H_{d}(0, t) \int_{-\infty}^{\infty}\left|H_{a}(j 2 \pi f, t)\right|\left|H_{b}(j 2 \pi f, t)\right|\left[\cos \left(\theta_{a}-\theta_{b}\right)\right. \\
\left.+j \sin \left(\theta_{a}-\theta_{b}\right)\right]\left[S_{x}(f)-S_{y}(f)\right] d f .
\end{array}
$$

It may be shown that $H(j 2 \pi f, t)$ has an imaginary part that is an odd function of frequency.

Using this fact, (14) becomes

$$
\begin{array}{r}
\bar{I}=H_{d}(0, t) \int_{-\infty}^{\infty}\left|H_{a}(j 2 \pi f, t)\right|\left|H_{b}(j 2 \pi f, t)\right|\left[\cos \left(\theta_{a}-\theta_{b}\right)\right] \\
{\left[S_{x}(f)-S_{y}(f)\right] d f .}
\end{array}
$$

Periodic signals such as CW signals or even periodic wide-sense random processes have power spectral densities expressed by means of the Dirac delta function, $\delta$ [Davenport, 1958; ch. 6]. For example, a CW signal, $V \cos \left(\omega_{0} t+\theta\right)$ has a power spectral density

$$
S_{c w}(f)=\frac{V^{2}}{4} \delta\left(f-f_{0}\right)+\frac{V^{2}}{4} \delta\left(f+f_{0}\right) .
$$

The total power, therefore, is

$$
\int_{-\infty}^{\infty} S_{c w}(f) d f=\frac{V^{2}}{2}
$$

as is well known. 
The output, $\bar{I}$, would become zero (a null would exist) when

$$
\begin{aligned}
\int_{-\infty}^{\infty} & \left|H_{a}(j 2 \pi f, t)\right|\left|H_{b}(j 2 \pi f, t)\right| \cos \left(\theta_{a}-\theta_{b}\right) S_{x}(f) d f \\
= & \int_{-\infty}^{\infty}\left|H_{a}(j 2 \pi f, t)\right|\left|H_{b}(j 2 \pi f, t)\right| \cos \left(\theta_{a}-\theta_{b}\right) S_{y}(f) d f .
\end{aligned}
$$

It is of considerable practical importance to consider those cases for which a null is obtained independent of the variations in the amplifiers and filter response functions. Since (18) contains no filter response function, the null is independent of the filter characteristics. From (18) it is also obvious that if the spectral densities of the $x(t)$ and $y(t)$ signals were equal over the amplifier pass bands, that is, if

$$
S_{x}(f)=S_{y}(f),
$$

a null is then obtained regardless of the time varying aspects of the amplifiers. Thus the important result is obtained that a drift or variation in the gain or phase response of the amplifiers and filter would not influence the null condition.

Further, if the amplifiers were constructed so that the frequency response determining components were stable, the amplifier characteristics could be written

$$
\begin{aligned}
& \left|H_{a}(j 2 \pi f, t)\right|\left|H_{b}(j 2 \pi f, t)\right| \cos \left(\theta_{a}-\theta_{b}\right) \\
& \quad=G_{a}(t) G_{b}(t)\left|H_{a}^{\prime}(j 2 \pi f)\right|\left|H_{b}^{\prime}(j 2 \pi f)\right| \cos \left(\theta_{a}-\theta_{b}\right)
\end{aligned}
$$

where $G_{a}(t)$ and $G_{b}(t)$ represent the time variable or unstable parts of the gains of the respective amplifiers and the primed H's are new time-stable frequency-dependent parts of the amplifier gains. The $\theta$ 's are now also independent of time.

The output of the system may then be written, since $G_{a}(t) G_{b}(t)$ is independent of $f$ over the bandpass,

$$
\begin{array}{r}
\bar{I}=H_{d}(0, t) G_{a}(t) G_{b}(t) \int_{-\infty}^{\infty}\left|H_{a}^{\prime}(j 2 \pi f)\right|\left|H_{b}^{\prime}(j 2 \pi f)\right| \\
\cos \left(\theta_{a}-\theta_{b}\right)\left[S_{x}(f)-S_{y}(f)\right] d f .
\end{array}
$$

This becomes zero when

$$
\begin{aligned}
& \int_{-\infty}^{\infty}\left|H_{a}^{\prime}(j 2 \pi f)\right|\left|H_{b}^{\prime}(j 2 \pi f)\right| \cos \left(\theta_{a}-\theta_{b}\right) S_{x}(f) d f \\
& =\int_{-\infty}^{\infty}\left|H_{a}^{\prime}(j 2 \pi f)\right|\left|H_{b}^{\prime}(j 2 \pi f)\right| \cos \left(\theta_{a}-\theta_{b}\right) S_{y}(f) d f
\end{aligned}
$$

and is independent of the amplifier drift or variation in gain. Such amplifiers are not too hard to realize by making the unstable frequency-dependent parts with pass bands broad compared to the stable frequency-dependent parts.
With such amplifiers a time-stable null condition expressed by (22) may be met with signals havingspectral densities with widely different frequency characteristics. For example $y(t)$ may be a sinusoidal signal

$$
y(t)=Y_{0} \cos \left(\omega_{0} t+\theta\right) .
$$

Then (22) becomes

$$
\begin{aligned}
& \int_{-\infty}^{\infty} \mid H_{a}^{\prime}(j 2 \pi f)|| H_{b}^{\prime}(j 2 \pi f) \mid \cos \left(\theta_{a}-\theta_{b}\right) S_{x}(f) d f \\
&= \int_{-\infty}^{\infty}\left[\left|H_{a}^{\prime}(j 2 \pi f)\right|\left|H_{b}^{\prime}(j 2 \pi f)\right| \cos \left(\theta_{a}-\theta_{b}\right)\right] \\
& {\left[\frac{Y_{0}^{2}}{4} \delta\left(f-f_{0}\right)+\frac{Y_{0}^{2}}{4} \delta\left(f+f_{0}\right)\right] d f } \\
&=\left|H_{a}^{\prime}\left(j 2 \pi f_{0}\right)\right|\left|H_{b}^{\prime}\left(j 2 \pi f_{0}\right)\right| \cos \left[\theta_{a}\left(f_{0}\right)-\theta_{b}\left(f_{0}\right)\right] \frac{Y_{0}^{2}}{2} .
\end{aligned}
$$

This is again independent of the amplifier gain variation.

\section{Measurement of Spectral Densities}

From the foregoing relationships, spectral densities of one source may be obtained in terms of that of another source.

Assume, for example, the value $S_{y}(f)$ is known and is also known to be essentially constant over the pass band, and it is desired to obtain the value of $S_{x}(f)$ which is also known to be constant over the pass band.

With reference to (18), a null condition is obtained when $S_{x}(f)=S_{y}(f)$. The two spectral densities are thus equated.

Let two different $x(t)$ signals be measured. Then

$$
\begin{aligned}
& S_{x 1}(f)=S_{y 1}(f) \\
& S_{x 2}(f)=S_{y 2}(f) .
\end{aligned}
$$

The ratio

$$
\frac{S_{y 2}(f)}{S_{y 1}(f)}=A
$$

may be measured by means of the precision attenuator (see fig. 1), and hence

$$
S_{x 2}(f)=A S_{x 1}(f) .
$$

In this case $S_{x 1}(f)$ and $S_{x 2}(f)$ need not be equal and only relative values of $S_{y}(f)$ are needed. The relative values of $S_{y}(f)$ may be obtained by means of the attenuator.

A CW signal could just as well be used in place of $S_{y}(f)$. Then

$$
S_{x 2}(f)=\frac{V_{2}^{2}}{V_{1}^{2}} S_{x 1}(f)=A S_{x 1}(f)
$$


where

$$
A=\frac{V_{2}^{2}}{V_{1}^{2}}
$$

and is again measured by the attenuator.

If $S_{x 2}(f)$ and $S_{x 1}(f)$ vary differently with frequency, the relationship between them may be obtained by using (22) to relate $S_{x 1}(f)$ with $S_{y 1}(f)$ and $S_{x 2}(f)$ with $S_{y 2}(f)$. Noting also that $S_{y 2}(f)=A S_{y 1}(f)$, the desired relationship becomes

$$
\begin{aligned}
\int_{-\infty}^{\infty} & \left|H_{a}^{\prime}(j 2 \pi f)\right|\left|H_{b}^{\prime}(j 2 \pi f)\right| \cos \left(\theta_{a}-\theta_{b}\right) S_{x 2}(f) d f \\
& =A \int_{-\infty}^{\infty}\left|H_{a}^{\prime}(j 2 \pi f)\right|\left|H_{b}^{\prime}(j 2 \pi f)\right| \cos \left(\theta_{a}-\theta_{b}\right) S_{x 1}(f) d f .
\end{aligned}
$$

If the spectral density of $S_{x 1}(f)$ is constant in value over the pass band, (30) becomes

$$
\begin{aligned}
& \int_{-\infty}^{\infty}\left|H_{a}^{\prime}(j 2 \pi f)\right|\left|H_{b}^{\prime}(j 2 \pi f)\right| \cos \left(\theta_{a}-\theta_{b}\right) S_{x 2}(f) d f \\
& \int_{-\infty}^{\infty}\left|H_{a}^{\prime}(j 2 \pi f)\right|\left|H_{b}^{\prime}(j 2 \pi f)\right| \cos \left(\theta_{a}-\theta_{b}\right) d f \\
& =A S_{x 1}(f) \text {. }
\end{aligned}
$$

In practice, this is what is usually measured. This gives the equivalent constant spectral density that would yield the same total power as the actual spectral density function when both are integrated over the actual pass band response function. This means the relative pass band response function of the actual system used should be given when stating the equivalent $S_{x}(f)$.

A system that meets the conditions imposed by (22) lends itself very well to a measurement of the relative pass band response function,

$$
K\left|H_{a}^{\prime}(j 2 \pi f)\right|\left|H_{b}^{\prime}(j 2 \pi f)\right| \cos \left(\theta_{a}-\theta_{b}\right) .
$$

Here $K$ is a constant and represents the fact that only relative and not absolute values are needed as a function of frequency. The above function is obtained by using CW signals for both the $x(t)$ and $y(t)$ signals. The $y(t)$ signal may be varied in level to maintain a null as $x(t)$ is varied in frequency but kept at a constant level. The relative values of the attenuator setting as a function of frequency give the desired relationship.

Other ways of using the system may readily come to mind. One other will be briefly mentioned. Another uncorrelated signal $v(t)$ with spectral density $S_{v}(f)$ may be combined with the $x(t)$ signal. The relative amounts of this extra signal may be measured by an attenuator external to the $x(t)$ signal in much the same way as is done with the $y(t)$ signal. In this case, the $y(t)$ signal need not be varied and, (19) could be written

$$
\begin{aligned}
S_{y}(f)=S_{x 1}(f) & +S_{v 1}(f) \\
= & S_{x 2}(f)+S_{v 2}(f)=S_{x 3}(f)+S_{v 3}(f) .
\end{aligned}
$$

If only relative values of $S_{v}(f)$ are known a useful relation is

where

$$
\frac{S_{x 3}(f)}{S_{x 1}(f)}=1+\left(\frac{S_{x 2}}{S_{x 1}}-1\right)\left(\frac{A_{31}-1}{A_{21}-1}\right)
$$

$$
\begin{aligned}
A_{31} & =\frac{S_{v 3}}{S_{v 1}} \\
A_{21} & =\frac{S_{v 2}}{S_{v 1}} .
\end{aligned}
$$

\subsection{Broad Spectral Signals Versus CW Signals}

As indicated, either broad spectral signals or a CW signal may be used as the $y(t)$ signal to compare various random $x(t)$ signals. When $\mathrm{CW}$ signals are used, it is important that the frequency of the CW signal be constant. Also, practical usage dictates finite averaging times at the output. This means that a CW $y(t)$ signal would produce less random fluctuation than a random $y(t)$ signal.

Broad spectral $y(t)$ signals, however, place less stringent requirements on the constancy of the band pass characteristics, especially if their spectral densities are similar to those of $x(t)$. A broad spectral nonrandom signal would produce less random fluctuation than a random signal and still have those advantages of broad spectral signals.

\subsection{Residual Noise}

If the signals $z_{a}(t)$ and $z_{b}(t)$ are correlated an additional term

$$
\left|H_{a}(j 2 \pi f, t)\right|\left|H_{b}(j 2 \pi f, t)\right|\left|S_{z_{a} z_{b}}(f)\right| \cos \left(\theta_{a}-\theta_{b}+\phi_{z_{a} z_{b}}\right)
$$

would have occurred. Here, $\phi_{z_{a} z_{b}}$ is the relative phase of the correlated part of the amplifier noise signal in (15). This represents a contribution in the output due to correlation between the two amplifier noise signals. While there is usually very little correlation between the two amplifier noise signals there can be contributions by the two amplifier noises that have common origins and are therefore correlated. Whether the effect of this correlated residual noise can be ignored depends on the relative strength of signals being measured to the residual noise and the desired accuracy of measurement.

This residual noise may possibly be reduced by proper design of the amplifier, or by using special networks that prevent coupling or that cause shifts in the phase of the correlated parts of the residual noise such that the quantity $\theta_{a}-\theta_{b}+\phi_{z_{a^{z}} b}$ in (17) equals $\pi / 2$, thus making the cosine factor zero.

Also, the residual noise may be simply measured by the use of two sources of different known levels. The principle may be simply demonstrated by assuming (this is not a necessary assumption) all signals have constant spectral densities within the amplifier pass bands and the amplifiers have equal phase shifts $\left(\theta_{a}=\theta_{b}\right)$. Using (15) with the assumption that no signals are correlated except $z_{a}(t)$ and 
$z_{b}(t)$ and that the null condition holds, then

$$
S_{x}(f)-S_{y}(f)+\left|S_{z_{a} z_{b}}(f)\right| \cos \phi_{z_{a} z_{b}}=0 .
$$

For two different levels of $S_{x}(f)$, this yields

$$
\left|S_{z_{a^{z} b}}(f)\right| \cos \phi_{z_{a^{z} b}}=\frac{A S_{x 1}-S_{x 2}}{1-A}
$$

where

$$
A=\frac{S_{y 2}(f)}{S_{y 1}(f)}
$$

\section{Relative Error of a Single Measurement}

Any practical system will average the output over a finite time. Because this time is finite, random fluctuations will appear in the output giving rise to errors when the output indicator is read.

For simplicity in evaluating this error, the following assumptions in addition to those above (with one exception) will be made.

1. Each amplifier has a square band pass of width B.

2 . The random processes giving rise to $x(t), z_{a}(t)$, and $z_{b}(t)$ are considered to be Gaussian, and the respective signals are independent and thus uncorrelated. Also, the various signal sources have zero means.

3. The signal $x(t)$ has a spectral density $S_{x}(f)$ that is constant over the band.

4. The signal, $y(t)=Y_{0} \cos \left(\omega_{0} t\right)$, is now a deterministic (nonrandom) CW signal centered in the pass band of the amplifier. The former theory still applies. The present system merely represents sample functions of the ensemble with time origins fixed relative to the $\mathrm{CW}$ signal.

5. The two amplifier noise signals, $z_{a}(t)$ and $z_{b}(t)$, are noncorrelated. 'Their respective spectral densities $S_{z_{a}}(f)$ and $S_{z_{b}}(f)$ are constant over the band.

6. The exception to the former assumptions is that the response functions of the amplifiers and filter are constant with time.

It is quite common to represent a narrow band of noise of band width $B$ around a central frequency by [Davenport, p. 158]

$$
\begin{aligned}
X(t) & =X_{c}(t) \cos \omega_{0} t-X_{s}(t) \sin \omega_{0} t \\
& =R(t) \cos \left[\omega_{0} t-\phi(t)\right]
\end{aligned}
$$

where $X_{c}(t), X_{s}(t), R(t)$, and $\phi(t)$ are random fluctuations that are slowly varying (due to the narrow band) with respect to the central frequency, $f_{0}=$ $\omega_{0} / 2 \pi$. Similarly

$$
\begin{aligned}
& z_{a}(t)=Z_{a c}(t) \cos \omega_{0} t-Z_{a s}(t) \sin \omega_{0} t \\
& z_{b}(t)=Z_{b c}(t) \cos \omega_{0} t-Z_{b s}(t) \sin \omega_{0} t .
\end{aligned}
$$

It can be shown [Davenport, p. 158] that

$$
\overline{X_{c}^{2}}=\overline{X_{s}^{2}}=2 \int_{0}^{\infty} S_{x}(f) d f=2 S_{x}(0) B
$$

$$
\begin{gathered}
\overline{Z_{a c}^{2}}=\overline{Z_{a s}^{2}}=2 S_{z_{a}}(0) B \\
\overline{Z_{b c}^{2}}=\overline{Z_{b s}^{2}}=2 S_{z_{b}}(\text { ) } B \\
\left.\overline{X_{c} X_{s}}=\overline{Z_{a c} Z_{a s}}=\overline{Z_{b c} Z_{b s}}=\right)
\end{gathered}
$$

where $S_{x}(0)$ denotes the constancy of $S_{x}$ with frequency.

Also, it can be shown [van der Ziel, 1954] that

where

$$
\overline{X_{c}^{2}(t) X_{c}^{2}(t+\tau)}=2 R_{x c}^{2}(\tau)+\overline{\left(X_{c}^{2}\right)^{2}}
$$

$$
\begin{array}{r}
R_{x c}(\tau)=\overline{X_{c}(t) X_{c}(t+\tau)}=\int_{-\infty}^{\infty} S_{x}(f) e^{j\left(\omega-\omega_{0}\right) \tau} d f \\
=2 S_{x}(0) B \frac{\sin \pi B \tau}{\pi B \tau}
\end{array}
$$

since $S_{x}(0)$ is constant. Similar relationships hold for $X_{s}(t), Z_{c}(t)$, and $Z_{s}(t)$.

It will also be assumed that the two amplifier noise signals are of equal strength. Hence

$$
S_{z_{a}}(0)=S_{z_{b}}(0) \equiv S_{z}(0) \text {. }
$$

The inputs to the two amplifiers will be Input $a$

$e_{a}(t)=\left[X_{c}(t)+Y_{0}+Z_{a c}(t)\right] \cos \omega_{0} t$

Input $b$

$$
-\left[X_{s}(t)+Z_{a s}(t)\right] \sin \omega_{0} t
$$

$e_{b}(t)=\left[X_{c}(t)-Y_{0}+Z_{b c}(t)\right] \cos \omega_{0} t$

$$
-\left[X_{s}(t)+Z_{b s}(t)\right] \sin \omega_{0} t .
$$

For mathematical convenience, the filter is considered to consist of two parts; (1) a zonal filter (i.e., a filter that has a system function that is unity over the pass band and zero elsewhere) that effectively integrates [Freeman, p. 226] the output of the multiplier over a period long compared to that of the central frequency $f_{0}$, and $(2)$ a filter that is effective at much lower frequencies.

The output of the multiplier, $M$, is effectively time averaged over a cycle of the center frequency, $\omega_{0}$, by the zonal filter. Thus

$$
\begin{array}{r}
M(t)=<e_{a}(t) e_{b}(t)>=\frac{1}{2}\left\{\left[X_{c}(t)+Y_{0}+Z_{a c}(t)\right]\right. \\
{\left[X_{c}(t)-Y_{0}+Z_{b c}(t)\right]} \\
\left.+\left[X_{s}(t)+Z_{a s}(t)\right]\left[X_{s}(t)+Z_{b s}(t)\right]\right\}
\end{array}
$$

where $\left\langle e_{a}(t) e_{b}(t)\right\rangle$ represents the temporal average.

To obtain the spectral density of $M$, its auto- 
correlation function is needed. Now

$$
R_{M}(\tau)=\overline{M(t) M(t+\tau)}
$$

If the value of $M(t)$ and $M(t+\tau)$ are used as given in (48) together with other relationships expressed above, it may be shown that

$$
\begin{aligned}
& R_{M}(\tau)=\frac{1}{4}\left\{\frac { B ^ { 2 } \operatorname { s i n } ^ { 2 } \pi B \tau } { ( \pi B \tau ) ^ { 2 } } \left[16 S_{x}^{2}(0)\right.\right. \\
& \left.+16 S_{x}(0) S_{z}(0)+8 S_{z}^{2}(0)\right]+4 Y_{0}^{2} S_{z}(0) B \frac{\sin \pi B \tau}{\pi B \tau} \\
& \left.\quad+16 S_{x}^{2}(0) B^{2}-8 Y_{0}^{2} S_{x}(0) B+Y_{0}^{4}\right\} .
\end{aligned}
$$

Since $R_{M}(\tau)$ is an even function of $\tau$

$$
S_{M}(f)=2 \int_{0}^{\infty} R_{M}(\tau) \cos (2 \pi f \tau) d \tau
$$

Substituting the value of $R_{M}(\tau)$ as given by (50) and integrating

$$
S_{M}(f)=\frac{1}{4}\left[4 S_{x}(0) B-Y_{0}\right]^{2} \delta(f)
$$

$$
+\left\{\begin{aligned}
& Y_{0}^{2} S_{z}(0)+2\left[2 S_{x}^{2}(0)+2 S_{x}(0) S_{z}^{2}(0)\right. \\
&\left.+S_{z}^{2}(0)\right][B-|f|] \\
& \text { for } 0 \leq|f|<\frac{B}{2} \\
& 2\left[2 S_{x}^{2}(0)+2 S_{x}(0) S_{z}(0)+S_{z}^{2}(0)\right][B-|f|] \\
& \text { for } \frac{B}{2}<|f|<B \\
& 0 \text { for }|f| \geq B .
\end{aligned}\right.
$$

The mean value of the indicator deflection, $\bar{I}$, is given by

$$
\bar{I}=\frac{1}{2} H_{d}(0)\left[4 S_{x}(0) B-Y_{0}^{2}\right]
$$

which may be obtained from (48) by taking the average over the ensemble and multiplying by the d-c response function $H_{d}(0)$. The same value can be obtained by integrating (52) over the vicinity of zero frequency, taking the square root, and multiplying by $H_{d}(0)$. At null, of course, $\bar{I}$ is zero which occurs when

$$
Y_{0}^{2}=4 S_{x}(0) B .
$$

In adjusting the output to a null, the fluctuations in the output cause an uncertainty in the value of $Y_{0}$ for a null condition. This means that the measured value of $Y_{0}$ and consequently the measured value of $S_{x}(0)$ are random variables. These are designated $Y_{0 m}$ and $S_{z m}(0)$ respectively and are related through $(54)$; that is

$$
Y_{0 m}^{2}=4 S_{x m}(0) B \text {. }
$$

The relative error, $\epsilon$, in a single measurement is now defined as

$$
\begin{aligned}
\epsilon & =\frac{\sqrt{\left(S_{x m}(0)-\overline{S_{x m}(0)}\right)^{2}}}{\overline{S_{x m}(0)}} \\
& =\frac{\sqrt{\overline{\Delta S_{x m}^{2}}(0)}}{\overline{S_{x}(0)}}
\end{aligned}
$$

where

and

$$
S_{x m}(0)-\overline{S_{x m}(0)}=\Delta S_{x}(0)
$$

$$
\overline{S_{x m}(0)}=S_{x}(0) .
$$

It is assumed the operator (or servomechanism) in adjusting the attenuator makes an error, $\Delta Y$, (which is a random variable) that just compensates for the fluctuation, $\Delta I$, in $I(t)$, where

$$
\Delta I=I(t)-\bar{I}
$$

or

$$
\overline{\Delta I^{2}}=\overline{I^{2}(t)}-(\bar{I})^{2} .
$$

This means, using (53), that

$$
\Delta I=-\frac{1}{2} H_{d}(0)\left[4 S_{x}(0) B-\left(Y_{0}+\Delta Y\right)^{2}\right] .
$$

Simplifying by the use of (54)

$$
\Delta I=H_{d}(0) Y_{0} \Delta Y
$$

where $\Delta Y^{2}$ has been neglected compared to $\Delta Y Y_{0}$. Using (58) it can be shown that

$$
\overline{\Delta I^{2}}=\int_{-\infty}^{\infty}\left|H_{d}(j 2 \pi f)\right|^{2} S_{M}(f) d f-(\bar{I})^{2} .
$$

When $H_{d}(j 2 \pi f)$ is nonzero except at zero and nearzero frequencies, it follows that essentially

$$
\overline{\Delta I^{2}}=S_{M}^{\prime}(0) \int_{-\infty}^{\infty}\left|H_{d}(j 2 \pi f)\right|^{2} d f .
$$

Now, from (52), and using (54)

$$
S_{M}^{\prime}(0)=4 S_{x}^{2}(0) B\left[1+2 \gamma+\frac{\gamma^{2}}{2}\right]
$$

where

$$
\gamma=\frac{S_{z}(0)}{S_{x}(0)}
$$

and the prime denotes $S_{M}(0)$ less the term involving the delta function.

The quantity $1+\gamma$ is equivalent to the operating noise figure. 'This would reduce to the standard noise figure when $x(t)$ is equivalent to a signal that originates from a resistor at a temperature of $290^{\circ} \mathrm{K}$. Using various relations as given above, the follow- 
ing chain of relations may be obtained

$$
\begin{aligned}
\epsilon^{2} & =\frac{\overline{\Delta S_{x}^{2}(0)}}{S_{x}^{2}(0)}=\frac{\overline{4 \Delta Y^{2}}}{Y_{0}^{2}}=\frac{\overline{4 \Delta I^{2}}}{H_{d}^{2}(0) Y_{0}^{4}} \\
& =\frac{4}{H_{d}^{2}(0) Y_{0}^{4}}\left[\int_{-\infty}^{\infty}\left|H_{d}(j 2 \pi f)\right|^{2} S_{M}(f) d f-(\bar{I})^{2}\right] \\
& =\frac{16 S_{x}^{2}(0) B}{Y_{0}^{4}}\left(1+2 \gamma+\frac{\gamma^{2}}{2}\right) \int_{-\infty}^{\infty}\left|\frac{H_{d}(j 2 \pi f)}{H_{d}(0)}\right|^{2} d f \\
& =\frac{1}{B}\left(1+2 \gamma+\frac{\gamma^{2}}{2}\right) \int_{-\infty}^{\infty}\left|\frac{H_{d}(j 2 \pi f)}{H_{d}(0)}\right|^{2} d f .
\end{aligned}
$$

For a simple RC filter of time constant $T$, the integral is equal to $\frac{1}{2 T}$. For a critically damped system the integral would be $1 / 2$ that given above.

Using the simple RC filter, the equation for $\epsilon$ becomes

$$
\epsilon=\left(\frac{1}{2}+\gamma+\frac{1}{4} \gamma^{2}\right)^{1 / 2} \frac{1}{\sqrt{B T}} .
$$

For example, if

$$
\begin{aligned}
B & =5 \times 10^{3} \mathrm{c} / \mathrm{s} \\
\gamma & =1 \\
T & =3.5 \mathrm{sec}
\end{aligned}
$$

then

$$
\epsilon=10^{-2} \text { or } 1 \text { percent. }
$$

If $\gamma=10$, then $T$ must be increased to $80 \mathrm{sec}$ to obtain the same value of $\epsilon$. If $\gamma=0$, then $T$ need only be $1.0 \mathrm{sec}$ for the same value of $\epsilon$.

\section{Conclusions}

The system described has several desirable characteristics.

1. The normally used high-speed switch has been eliminated. This removes the problems of switch noise, insertion loss, and instability.

2. The effect of amplifier gain drift has been essentially eliminated.

3. The system is operated under null conditions and the indicator null position is therefore independent of amplifier gain settings.

4. A precision, continuously variable piston attenuator may be used.
5. Signals of widely varying strengths may be compared.

6. The system is capable of measuring its own effective pass band response to a high degree of precision.

7. Problems of impedance variations in a dissipative attenuator have been removed.

8. The system is quite insensitive to "hum" and other such signals that might possibly modulate the normally present signals. This follows since the null condition is independent of a time variable system response. This time variation if produced by "hum" or other signals is what often gives troubles in systems, especially those using linear or envelope detectors and switching frequencies commensurate with the undesirable modulating frequencies.

Some undesirable aspects are:

1. One must have two low noise amplifiers.

2. The signal power is now divided into two channels

3. A determination of the effect of correlation between the internal amplifiers may be necessary in some situations. This may be done by comparing two standard sources of different levels.

4. An appropriate multiplier is needed. Only the output at zero frequency is of interest and this fact removes many problems. Also, an ideal multiplier is not necessarily needed. The main consideration is that no output at zero frequency should exist when only one input is en ergized.

The author expresses his gratitude for the valuable discussions and encouragement given by M. C. Selby and M. G. Arthur.

\section{References}

Davenport, W. B., Jr., and W. L. Root, An Introduction to the Theory of Random Signals and Noise, ch. 6, p. 158 (McGraw-Hill Book Co., Inc., New York, N.Y., 1958). Dicke, R. H., The measurement of thermal radiation at microwave frequencies, Rev. Sci. Instr.17, No. 7, 268 (1946).

Fink, H. J., A new absolute noise thermometer at low temperatures, Can. J. Phys. 37, 1397 (1959).

Freeman, J. J., Principles of Noise, p. 226; p. 274 (John Wiley \& Sons, Inc., New York, N.Y., 1958).

van der Ziel, A., Noise, p. 312 (Prentice-Hall, Inc., Englewood Cliffs, N.J., 1954). 